\title{
Electronic and spin structure of the wide-band-gap topological insulator: Nearly stoichiometric $\mathrm{Bi}_{2} \mathrm{Te}_{2} \mathrm{~S}$
}

\author{
E. Annese, ${ }^{1,2, *}$ T. Okuda, ${ }^{2}$ E. F. Schwier, ${ }^{2}$ H. Iwasawa, ${ }^{2}$ K. Shimada, ${ }^{2}$ M. Natamane, ${ }^{2}$ M. Taniguchi, ${ }^{2}$ I. P. Rusinov, ${ }^{3,4}$ \\ S. V. Eremeev, ${ }^{5,3,4}$ K. A. Kokh,,${ }^{6,7}$ V. A. Golyashov, ${ }^{8,7,4}$ O. E. Tereshchenko, ${ }^{8,7,4}$ E. V. Chulkov, ${ }^{9,10,3,4}$ and A. Kimura ${ }^{11}$ \\ ${ }^{1}$ Centro Brasileiro de Pesquisas Físicas, rua Dr. Xavier Sigaud, 150 Urca, Rio de Janeiro, Brazil \\ ${ }^{2}$ Hiroshima Synchrotron Radiation Center, Hiroshima University, 2-313 Kagamiyama, Higashi-Hiroshima 739-0046, Japan \\ ${ }^{3}$ Tomsk State University, 634050 Tomsk, Russia \\ ${ }^{4}$ Saint Petersburg State University, 198504 Saint Petersburg, Russia \\ ${ }^{5}$ Institute of Strength Physics and Materials Science, 634021 Tomsk, Russia \\ ${ }^{6}$ Institute of Geology and Mineralogy, 630090 Novosibirsk, Russia \\ ${ }^{7}$ Novosibirsk State University, 636090 Novosibirsk, Russia \\ ${ }^{8}$ Institute of Semiconductor Physics, 636090 Novosibirsk, Russia \\ ${ }^{9}$ Donostia International Physics Center (DIPC), 20018 San Sebastián/Donostia, Basque Country, Spain \\ ${ }^{10}$ Departamento de Física de Materiales UPV/EHU, Centro de Física de Materiales CFM - MPC and Centro Mixto CSIC-UPV/EHU, \\ 20080 San Sebastián/Donostia, Basque Country, Spain \\ ${ }^{11}$ Graduate School of Science, Hiroshima University, 1-3-1 Kagamiyama, Higashi-Hiroshima 739-8526, Japan
}

(Received 22 November 2017; published 10 May 2018)

\begin{abstract}
We have grown the phase-homogeneous ternary compound with composition $\mathrm{Bi}_{2} \mathrm{Te}_{1.85} \mathrm{~S}_{1.15}$ very close to the stoichiometric $\mathrm{Bi}_{2} \mathrm{Te}_{2} \mathrm{~S}$. The measurements performed with spin- and angle-resolved photoelectron spectroscopy as well as density functional theory and $G W$ calculations revealed a wide-band-gap three-dimensional topological insulator phase. The surface electronic spectrum is characterized by the topological surface state (TSS) with Dirac point located above the valence band and Fermi level lying in the band gap. TSS band dispersion and constant energy contour manifest a weak warping effect near the Fermi level along with in-plane and out-of-plane spin polarization along the $\bar{\Gamma}-\bar{K}$ line. We identified four additional states at deeper binding energies with high in-plane spin polarization.
\end{abstract}

DOI: 10.1103/PhysRevB.97.205113

\section{INTRODUCTION}

Topological insulators (TIs) are characterized by an insulating energy gap in the bulk and the gapless spin-polarized metallic surface states with linear Dirac conelike dispersion in the energy-momentum space with helical spin textures protected by time-reversal symmetry, which can favor the quantum spin transport without heat dissipation [1,2].

The binary tetradymitelike $\mathrm{Bi}$ chalcogenides $\mathrm{Bi}_{2} \mathrm{Se}_{3}$ and $\mathrm{Bi}_{2} \mathrm{Te}_{3}$ are widely regarded as model examples of the threedimensional topological insulators since they host a single metallic Dirac state [1-7]. However, these compounds are not insulators, but have metallic bulk conductivity as a consequence of unintentional doping by defects: the Se vacancies in $\mathrm{Bi}_{2} \mathrm{Se}_{3}$ and $\mathrm{Bi}-\mathrm{Te}$ antisite defects in $\mathrm{Bi}_{2} \mathrm{Te}_{3}[4,5,8]$. The isolation of the surface states from the bulk electronic states and the Fermi level $\left(E_{F}\right)$ located in the bulk band gap is the minimal requirement for the quantum spin transport. To this end, various experimental efforts exploited the tune of $E_{F}$ with doping $[7,9]$. On the other hand, the dopants themselves could become a scattering source and therefore reduce spin lifetime [10]. One of the strategies to control the native defects in Teand Se-containing TIs is using ternary Bi-Te-Se alloy systems.

*emiliaannese@gmail.com
It was demonstrated that the solutions with Te-rich and Se-poor conditions minimize the defects and manifest a single Dirac cone that intersects the Fermi level $[8,10-13]$.

The electronic structure of tetradymite itself, $\mathrm{Bi}_{2} \mathrm{Te}_{2} \mathrm{~S}$, has been calculated in several works [14-16] alongside other ternary compounds. In contrast to $\mathrm{Bi}_{2}\left(\mathrm{Te}_{1-x} \mathrm{Se}_{x}\right)_{3}$ solid solutions, $\mathrm{Bi}_{2} \mathrm{Te}_{2} \mathrm{~S}$ is a separate compound of the $\mathrm{B}_{2} \mathrm{Te}_{3}-\mathrm{Bi}_{2} \mathrm{~S}_{3}$ system $[17,18]$. According to the recent review on natural tetradymites by Cook et al. [19], a homogeneity region for the compound seems to occupy a wide range of compositions (30$40 \mathrm{~mol} . \%$ of $\mathrm{Bi}_{2} \mathrm{~S}_{3}$ ). On the other hand, Glatz [20] has assumed that $\mathrm{Bi}_{2} \mathrm{Te}_{2} \mathrm{~S}$ forms two phases-an incongruent close-tostoichiometric (Te-rich) $\beta$ phase and a sulfur-rich $\gamma$ phase with congruent melting. The latter phase with composition of $\mathrm{Bi}_{2} \mathrm{Te}_{1.6} \mathrm{~S}_{1.4}$ and cell parameters of $a=4.196(4) \AA$ and $c=29.44(3) \AA$ A was studied by Ji et al. [21]. The angle-resolved photoemission spectroscopy (ARPES) measurements revealed that the S-rich phase is a three-dimensional (3D) TI with a band gap of $200 \mathrm{meV}$.

This work aims to provide a detailed characterization of the electronic and spin structures of topological and higher binding-energy (BE) surface states in the nearly stoichiometric $\mathrm{Bi}_{2} \mathrm{Te}_{2} \mathrm{~S}$ grown by a modified vertical Bridgman method. We show that the surface electronic spectrum of this compound is characterized by the topological surface state (TSS), which Dirac point is located above the valence band and Fermi level 
lying in the band gap. Owing to its remarkable electronic properties consisting of $\approx 400 \mathrm{meV}$ bulk band gap and weakly warped topological Dirac state, the $\mathrm{Bi}_{2} \mathrm{Te}_{2} \mathrm{~S}$ can be regarded as the model topological insulator to a greater extent than the extensively used $\mathrm{Bi}_{2} \mathrm{Se}_{3}$. Apart from the interesting property related to the TSS, the topological insulator manifests an electronic structure, which can be a fruitful playground for more traditional effects in electronic surface science: band bending near the surface, formation of the quantum well states, and the Rashba-split deep surface states.

\section{EXPERIMENTAL AND COMPUTATIONAL DETAILS}

The synthesis of charge was done by careful fusion of elementary $\mathrm{Bi}, \mathrm{Te}$, and $\mathrm{S}$ in a horizontal quartz ampoule. Then the charge was transferred into a carbon-coated ampoule with conical tip and recrystallized by the modified vertical Bridgman method [22,23]. At both stages, the ampoules were evacuated down to $10^{-3}$ torr before sealing. The samples cut from the obtained single-crystalline boule had a composition $\mathrm{Bi}_{2} \mathrm{Te}_{1.85} \mathrm{~S}_{1.15}$ according to energy-dispersive $\mathrm{x}$-ray chemical analysis. Powder diffraction data confirmed the tetradymite structure with cell parameters $a=4.241 \AA, c=29.578 \AA$ (Fig. 1). According to differential thermal analysis, the sample melts congruently at $\approx 595^{\circ} \mathrm{C}$. The obtained data do not allow unambiguous attribution of the grown crystal to the $\gamma$ or $\beta$ phase described in Ref. [20]. We state the production of a phase-homogeneous single-crystal material with a composition much closer to the stoichiometry of $\mathrm{Bi}_{2} \mathrm{Te}_{2} \mathrm{~S}$ than that studied in Ref. [21]. The measured in-plane Seebeck coefficient of the grown sample demonstrated $-250 \mu \mathrm{V} / \mathrm{K}$ at room temperature, suggesting an $n$-type conductivity. This value is comparable with the in-plane Seebeck coefficient of $\mathrm{Bi}_{2} \mathrm{Te}_{3}$, equal to $-280 \mu \mathrm{V} / \mathrm{K}$. The resistivity of the grown crystal showed a slightly higher value than for ternary compounds of the $\mathrm{Bi}_{2}\left(\mathrm{Te}_{1-x} \mathrm{Se}_{x}\right)_{3}$ series [13].

The ARPES experiment was performed at Laser-ARPES at the Hiroshima Synchrotron Radiation Center (HiSOR) using $s$-polarized light of $6.45 \mathrm{eV}$ and measured with a hemispherical analyzer (VG Scienta SES R4000) at $70 \mathrm{~K}$. The sample was cleaved in situ at room temperature in an ultrahigh vacuum below $1 \times 10^{-8} \mathrm{~Pa}$. The spin-resolved energy distribution

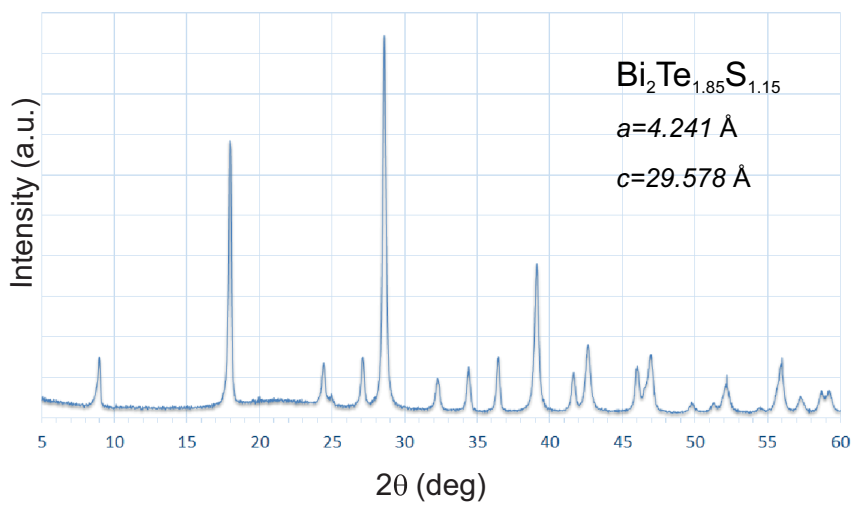

FIG. 1. X-ray powder diffraction pattern for $\mathrm{Bi}_{2} \mathrm{Te}_{1.85} \mathrm{~S}_{1.15}$ singlecrystal sample. curves (EDCs) are acquired with the efficient spin-resolved spectroscopy ESPRESSO end station at HiSOR [24,25] at 70 and $15 \mathrm{~K}$. An He $I_{\alpha}$ discharge lamp and $p$-polarized synchrotron light of $17 \mathrm{eV}$ are used as the excitation source for the spin-resolved photoemission experiment. The overall energy resolutions are set to $20 \mathrm{meV}$ at the spin-ARPES end station.

Electronic structure calculations were carried out within the density functional theory (DFT) and $G W$ approaches. The ground state has been obtained using the projector augmentedwave method [26,27] realized in the VASP code [28,29]. For this calculation, the Perdew, Burke, and Ernzerhof exchangecorrelation functional [30] with DFT-D3 van der Waals correction [31] was applied and the spin-orbit coupling was accounted in self-consistent electronic structure calculations. We applied $7 \times 7 \times 7 \Gamma$-centered $k$-point sampling of the Brillouin zone. The $G W$ correction has been calculated with a posteriori treatment of spin-orbit effects: this contribution is taken from the DFT calculations. Next, from the quasiparticle spectrum, we construct a bulk tight-binding model using the WANNIER90 code [32]. The chosen basis consists of six spinor $p$-type orbitals for each atom: $\left|p_{x}^{\uparrow}\right\rangle,\left|p_{y}^{\uparrow}\right\rangle,\left|p_{z}^{\uparrow}\right\rangle,\left|p_{x}^{\downarrow}\right\rangle$, $\left|p_{y}^{\downarrow}\right\rangle$, and $\left|p_{z}^{\downarrow}\right\rangle$. The low-lying $s$ orbitals are not taken into consideration. The surface tight-binding model is derived from the bulk one with the inclusion of band-bending effects obtained from surface calculations within DFT [33]. To study the electronic structure of $\mathrm{Bi}_{2} \mathrm{Te}_{1.85} \mathrm{~S}_{1.15}$, which differs from the stoichiometric compound, an extra $7.5 \%$ of sulfur is randomly distributed within each Te atomic layer. First, the bulk $G W$ calculations have been performed for the hypothetical $\mathrm{Bi}_{2} \mathrm{~S}_{3}$ with the same lattice parameters as for $\mathrm{Bi}_{2} \mathrm{Te}_{2} \mathrm{~S}$. Then we used the virtual crystal approximation to construct the tight-binding Hamiltonian for the bulk $\mathrm{Bi}_{2} \mathrm{Te}_{1.85} \mathrm{~S}_{1.15}$ as follows: $\hat{H}_{\mathrm{Bi}_{2} \mathrm{Te}_{1.85} \mathrm{~S}_{1.15}}=0.925 \hat{H}_{\mathrm{Bi}_{2} \mathrm{Te}_{2} \mathrm{~S}}+0.075 \hat{H}_{\mathrm{Bi}_{2} \mathrm{~S}_{3}}$. The bandbending effect in the surface tight-binding model for nearly stoichiometric $\mathrm{Bi}_{2} \mathrm{Te}_{1.85} \mathrm{~S}_{1.15}$ was taken to be the same as for the $\mathrm{Bi}_{2} \mathrm{Te}_{2} \mathrm{~S}$ compound.

\section{RESULTS AND DISCUSSIONS}

The DFT calculations [Fig. 2(a)] for stoichiometric $\mathrm{Bi}_{2} \mathrm{Te}_{2} \mathrm{~S}$ reproduce the results of earlier works $[14,15]$, demonstrating an indirect bulk gap of $\sim 300 \mathrm{meV}$ with the valence-band maximum (VBM) situated in the $\bar{\Gamma}-\bar{M}$ direction at $k_{\|}=$ $0.19 \AA^{-1}$ and spin-polarized topological surface state at $\bar{\Gamma}$ lying at 130 meV below VBM. It was shown earlier that the inclusion of many-body effects within the $G W$ approximation decreases the size of the bulk band gap at the Brillouin-zone center in the TI spectrum and increases the absolute band gap lowering the valence-band maximum [16,34-38], and even converting the indirect gap into the direct one in the case of $\mathrm{Bi}_{2} \mathrm{Se}_{3}[38,39]$. The calculated quasiparticle spectrum of $\mathrm{Bi}_{2} \mathrm{Te}_{2} \mathrm{~S}$ [Fig. 2(b)] demonstrates the increase of the indirect bulk band gap up to $390 \mathrm{meV}$ and reduction in the difference between the VBM and maximum of the valence band at $\bar{\Gamma}$ by $95 \mathrm{meV}$. Such changes in the bulk states also result in modification of the surface states and lead to pulling out the Dirac point above the VBM in accordance with experimental finding (which we show below). Accounting for the deviation of composition from stoichiometry does not lead to sizable modification of the bulk 

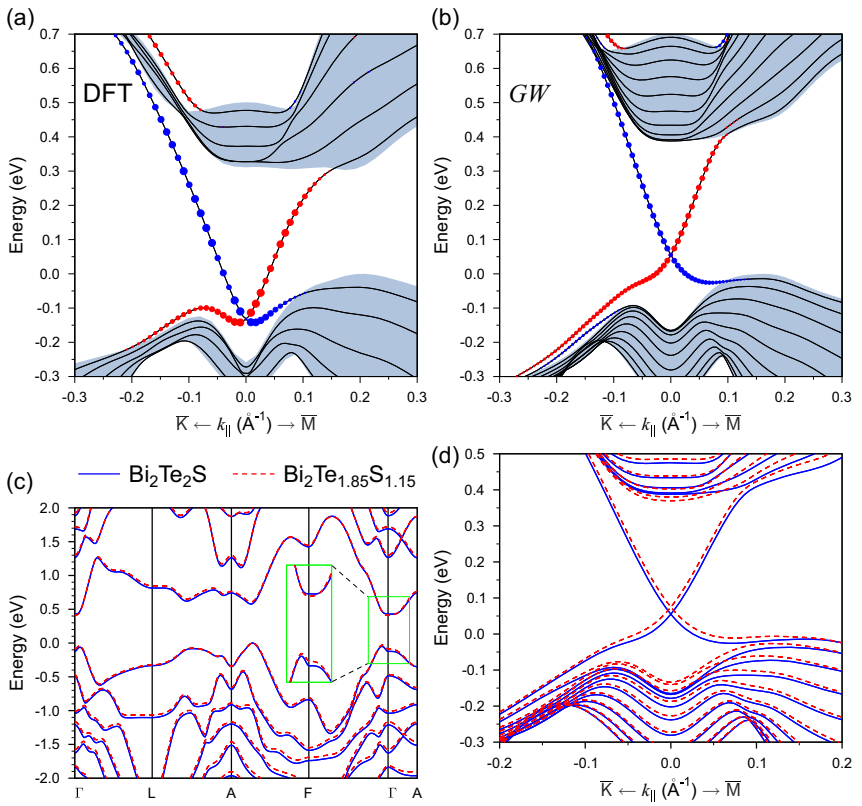

FIG. 2. The surface electronic spectrum of the $\mathrm{Bi}_{2} \mathrm{Te}_{2} \mathrm{~S}$ calculated within the (a) DFT and (b) $G W$ approaches. The comparison of the $\mathrm{Bi}_{2} \mathrm{Te}_{2} \mathrm{~S}$ and $\mathrm{Bi}_{2} \mathrm{Te}_{1.85} \mathrm{~S}_{1.15} G W$ spectra for (c) bulk and (d) surface.

band spectrum [see Fig. 2(c)]. The main change introduced by the appearance of an extra sulfur on the tellurium layers of a quintuple layer block is the reduction of the $\Gamma$ gap (by $54 \mathrm{meV}$ ), while the absolute gap is reduced by $20 \mathrm{meV}$ only. The resulting bulk gap of $370 \mathrm{meV}$ is more than $100 \mathrm{meV}$ larger than the

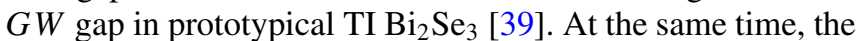
deviation from stoichiometry does not change the dispersion of the TSS and only moves up the Dirac point by $19 \mathrm{meV}$ [Fig. 2(d)]. Since the small deviation from the stoichiometric composition does not lead to any noticeable changes in the bulk and surface electronic spectra, below, for simplicity, we will call the grown near-stoichiometric compound as $\mathrm{Bi}_{2} \mathrm{Te}_{2} \mathrm{~S}$.

Figure 3 shows the experimental electronic structure close to $E_{F}$ on $\mathrm{Bi}_{2} \mathrm{Te}_{2} \mathrm{~S}$ along the $\bar{\Gamma}-\bar{M}$ direction. Measurements were performed at different photon energies $(6.45,17$, and $21 \mathrm{eV}$ ) and light polarization ( $s, p$, and unpolarized light) at $70 \mathrm{~K}$, except for the spectra at $17 \mathrm{eV}$ obtained at $15 \mathrm{~K}$. Variation of the photon energy leads to a modulation of the
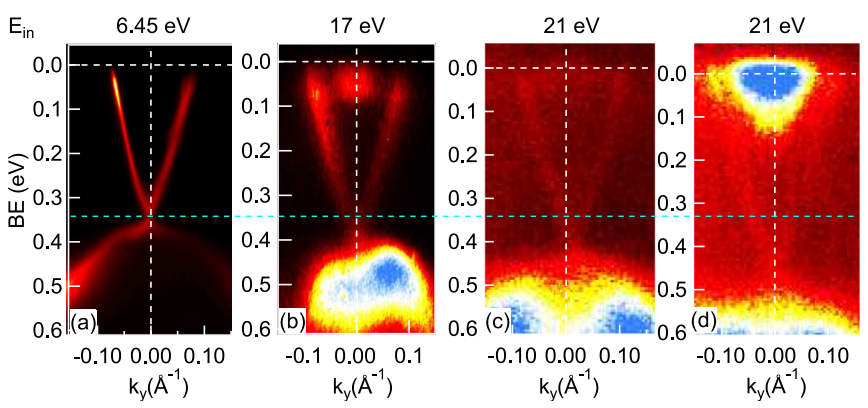

FIG. 3. ARPES spectra measured along $\bar{\Gamma}-\bar{M}$ with different photon energies: (a) $6.45 \mathrm{eV}$ ( $s$ polarization) at $70 \mathrm{~K}$, (b) $17 \mathrm{eV}(p$ polarization) at $15 \mathrm{~K}$, and (c),(d) $21.2 \mathrm{eV}$ at $70 \mathrm{~K}$. (d) Measurements on the same sample after $1 \mathrm{~h}$ of exposure to the discharge He lamp.
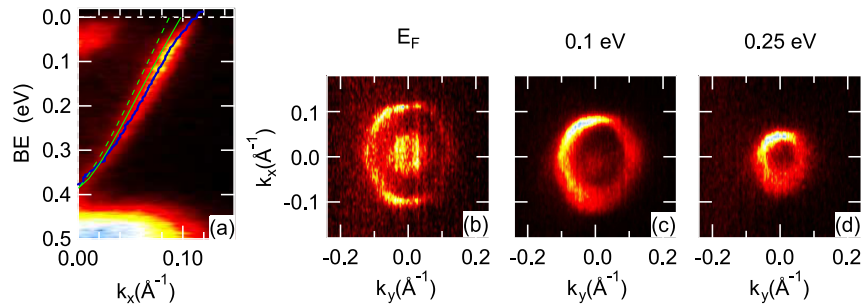

FIG. 4. (a) $E(k)$ photoemission map along $\bar{\Gamma}-\bar{K}$. The solid blue curve is a guide for the eyes; the solid and dashed green curves are dispersions of the TSS calculated within the DFT and $G W$ approaches, respectively. (b),(c) Constant energy contours of the topological surface state at different binding energies from 0 to $0.25 \mathrm{eV}$. Measurements were performed with $p$-polarized light at $15 \mathrm{~K}$.

photoemission intensity for both the topological surface state and the valence-band states [Figs. 3(a)-3(d)]. Figure 3(a) displays a single Dirac cone. The Dirac point (DP) and VBM are visible at binding energy of 0.34 and $0.36 \mathrm{eV}$, respectively. As far as the conduction band is not visible in the ARPES spectrum, we can estimate the bulk gap as of $\approx 400 \mathrm{meV}$, which is in fine agreement with the calculation result and is twice as large as the bulk gap reported for $\mathrm{Bi}_{2} \mathrm{Te}_{1.6} \mathrm{~S}_{1.4}$ (200 meV) [21].

A different position of the Dirac point is observed by changing the photon source. A fresh sample exposed to synchrotron light does not exhibit any shift of the Dirac point, which is always present in case of $\mathrm{He} I_{\alpha}$ unpolarized light excitation. The position of the Dirac point shifts rigidly towards higher binding energy [Figs. 3(c) and 3(d)] and a two-dimensional electron gas appears at the Fermi level [Fig. 3(d)]. The binding energy of the Dirac point saturates after $1 \mathrm{~h}$ exposure to an He discharge lamp. Similar behavior is observed for other topological insulators originating from the band-bending effect as a consequence of the $n$-type doping provided by adsorption of residual gases [40-42].

Figure 4(a) shows band energy dispersion along the $\bar{\Gamma}-\bar{K}$ direction. Although the $G W$ calculation results are in perfect agreement with experiment in part of the bulk gap and the Dirac-point position, the DFT calculation reproduces the experimental TSS slope slightly better [see the green solid and dashed curves in Fig. 4(a)]. The constant energy contours of the TSS evolve from the hexagonal to a circular shape in going from binding energy of 0.25 to $0 \mathrm{eV}$ [Figs. 4(b) and 4(c)], indicating a weak warping effect $[12,43,44]$.

We estimated the energy of the Dirac point, the effective mass $m^{*} / m$ of the TSS, the Dirac velocity $v$, and the warping strength coefficient $\lambda$ by analyzing the photoemission data according to Refs. [43,44], as reported in Table I. As can be seen from the table, the TSS of $\mathrm{Bi}_{2} \mathrm{Te}_{2} \mathrm{~S}$ has the warping comparable with that in $\mathrm{Bi}_{2} \mathrm{Se}_{3}$ and a Fermi velocity in between those in prototypical TIs $\mathrm{Bi}_{2} \mathrm{Te}_{3}$ and $\mathrm{Bi}_{2} \mathrm{Se}_{3}$.

Representative spin-resolved EDCs for in-plane and outof-plane components were measured at different $k$ points, as displayed in Fig. 5. $\mathrm{Bi}_{2} \mathrm{Te}_{2} \mathrm{~S}$ has a sizable in-plane and out-of-plane spin component (50\% and $20 \%$, respectively), and the up and down spins are reversed between opposite $k$ points along the $\bar{\Gamma}-\bar{K}$ and $\bar{\Gamma}-\bar{M}$ cuts. This is in agreement with previous results on binary and ternary topological insulators 
TABLE I. $m^{*} / m, v$, and $\lambda$ obtained from the analysis of the ARPES data. Values for $\mathrm{Bi}_{2} \mathrm{Te}_{3}$ and $\mathrm{Bi}_{2} \mathrm{Se}_{3}$ are taken from Ref. [44].

\begin{tabular}{lccc}
\hline \hline & $\mathrm{Bi}_{2} \mathrm{Te}_{2} \mathrm{~S}$ & $\mathrm{Bi}_{2} \mathrm{Te}_{3}$ & $\mathrm{Bi}_{2} \mathrm{Se}_{3}$ \\
\hline $\mathrm{DP}(\mathrm{eV})$ & 0.34 & 0.29 & 0.33 \\
$m^{*} / m$ & 0.19 & -0.3 & 0.3 \\
$v(\mathrm{eV} \AA)$ & 3.2 & 3.8 & 2.1 \\
$\lambda\left(\mathrm{eV}^{-3}\right)$ & 60 & 210 & 50 \\
\hline \hline
\end{tabular}

where an out-of-plane spin polarization is concomitant with TSS hexagonal warping $[12,43,44]$.

Figure 6(a) shows the ARPES spectra for deep-lying states taken at $\mathrm{He} I_{\alpha}$ along the $\bar{\Gamma}-\bar{K}$ direction. The characteristic features of the photoemission data are (i) two upward-convex parabolic states $S_{1}$ centered at $\pm 0.11 \AA^{-1}$, with maximum at $\mathrm{BE}=0.7 \mathrm{eV}$ and separated in two subbands for $k \geqslant|0.25|$ $\AA^{-1}$; (ii) the bright feature $S_{2}$ at $B E=1.23 \mathrm{eV}$; (iii) an upwardconvex arc $\mathrm{S}_{3}$ with binding-energy maximum at $1.75 \mathrm{eV}$ at $k=0$; (iv) two upward-convex parabolic states $\mathrm{S}_{4}$ centered at $\pm 0.15 \AA^{-1}$, with maximum at $\mathrm{BE}=2.3 \mathrm{eV}$ and crossing point at $\mathrm{BE}=2.6 \mathrm{eV}$. Figure 6(b) shows the calculated spectrum. The comparison with the calculations allows us to determine the nature of each state appearing in the photoemission map. The $S_{1}$ state can be assigned to the Rashba-split state, residing near the bottom of the first local valence-band gap, which is typical for tetradymitelike TIs [45]. It manifests as a peculiar band deviating from typical Rashba-split states by the suppression of the inner Rashba-split band in the vicinity of the Brillouin-zone center due to hybridization with bulk states. The bright feature at $\mathrm{BE}=1.23 \mathrm{eV}\left(\mathrm{S}_{2}\right)$ in the photoemission map derives from the spin-split band residing in the second local valence-band gap at $\approx-0.7 \mathrm{eV}$ [Fig. 6(b)]. The wide $\operatorname{arc} S_{3}$ can be assigned to the fourth bulk valence band in

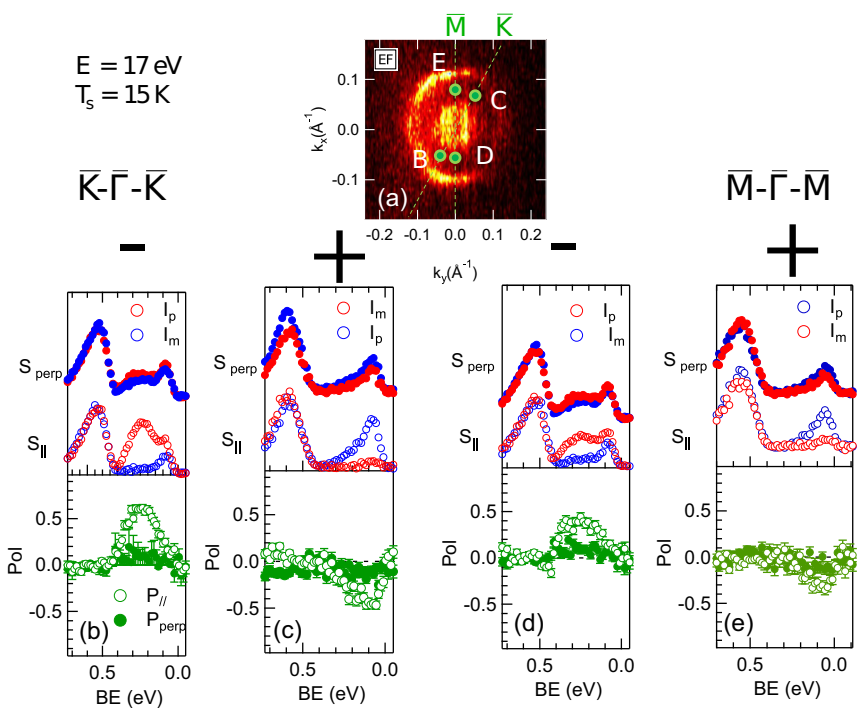

FIG. 5. (a) The constant energy contour of topological surface states at $E_{F}$. (b)-(e) Spin-resolved EDCs along $\bar{\Gamma}-\bar{K}$ and $\bar{\Gamma}-\bar{M}$ in $k$ space as identified by the letters in (a). Measurements were performed with $p$-polarized light of $17 \mathrm{eV}$ at $15 \mathrm{~K}$.
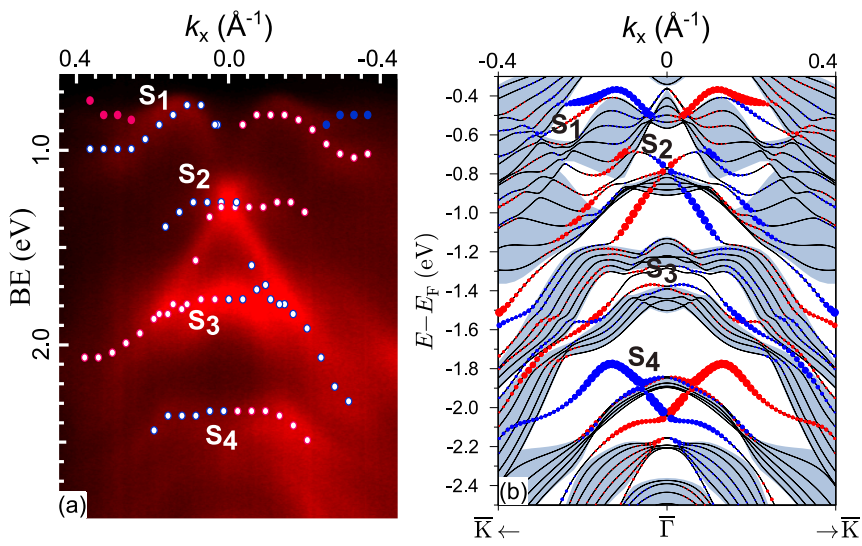

FIG. 6. (a) $E$ (k) photoemission map along $\bar{\Gamma}-\bar{K}$. (b) Calculated spin-resolved surface electronic structure with in-plane positive and negative spin components marked by red and blue circles, respectively.

which a resonant state resides. Finally, the deepest feature in the ARPES spectrum, $\mathrm{S}_{4}$, derives from the surface states located in the fourth and fifth local valence-band gaps visible in Fig. 6(b) in the energy window of $-1.8-2.2 \mathrm{eV}$. $\mathrm{S}_{4}$ presents typical Rashba-like splitting with Rashba energy $E_{\mathrm{R}}=265 \mathrm{meV}$ and the momentum offset $k_{\mathrm{R}}=0.13 \AA^{-1}$.

To unravel the spin structure of higher binding-energy states, we performed the spin-ARPES measurement, excited with unpolarized $\mathrm{He} I_{\alpha}$ light. Spin-up $I^{\uparrow}$ (red) and spin-down $I^{\downarrow}$ (blue) energy distribution curves as well as spin polarization $P$ (green curves with error bar) are represented in the top panels of Fig. 7. Figures 7(a) and 7(b) represent, respectively, the in-plane and out-of-plane components of spin polarization $\vec{P}$ ( $X$ and $Z$ in the spin detector coordinates as defined in Ref. [25]) along the $\bar{\Gamma}-\bar{K}$ direction, and some selected spectra for the in-plane and out-of-plane components along the $\bar{\Gamma}-\bar{M}$ direction are shown in Figs. 7(c) and 7(d). Spin-polarization curves were calculated as $P=\frac{\left(I^{\uparrow}-I^{\downarrow}\right)}{\left(I^{\uparrow}+I^{\downarrow}\right)} \cdot \frac{1}{S_{\text {eff }}}$, where $S_{\text {eff }}$ is the analyzer Sherman function. In Fig. 7(a), guide lines are drawn to highlight the $\mathrm{S}_{1}-\mathrm{S}_{4}$ electronic states. At the $\bar{\Gamma}$ point, a null in-plane and out-of-plane spin polarization within the experimental error bar are observed for all the features $(\mathrm{BE}$ $=1,1.45,2$, and $2.4 \mathrm{eV}$ ). Figures 7(e) and 7(f) show the resulting spin-polarization maps for the $P_{\text {in-plane }}$ and $P_{\text {out-of-plane }}$ spin components, respectively, along the $\bar{\Gamma}-\bar{K}$ direction. The $P_{\text {in-plane }}\left(P_{\text {out-of-plane }}\right)$ image is obtained by merging the polarization curves extracted from the analysis of EDCs measured in the $k$ interval of $[-0.36,0.36] \AA^{-1}$ with a step of 0.036 $\AA^{-1}$ [Figs. 7(a) and 7(b)]. The full data set acquisition of the $P_{\text {out-of-plane }}\left(P_{\text {in-plane }}\right)$ image took $12 \mathrm{~h}$. As can be seen in Fig. 7(e), all the bands $S_{1}-S_{4}$ demonstrate the in-plane spin orientation antisymmetric with respect to the $\bar{\Gamma}$ point. At the same time, the $S_{3}$ band manifests the spin polarization opposite to the other states. The extent of spin polarization of the $S_{2}$ state is smaller than the others, as follows from the low contrast in the in-plane polarization image. For $k_{x} \geqslant|0.25| \AA^{-1}$, the upper part of the $S_{1}$ band shows out-of-plane spin polarization as well as $S_{3}$ band [Fig. 7(f)]. 


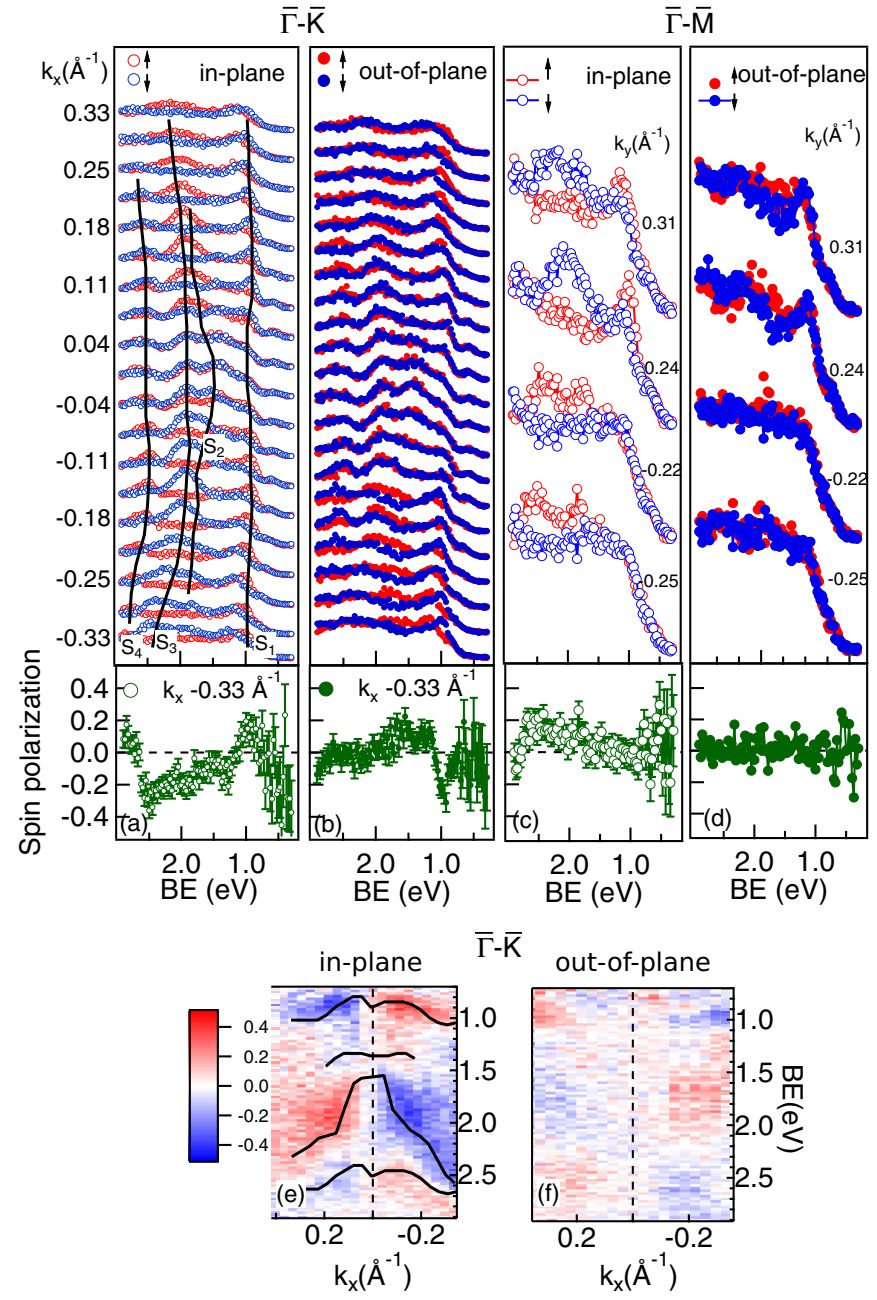

FIG. 7. (a) In-plane and (b) out-of-plane spin-resolved EDCs along the $\bar{\Gamma}-\bar{K}$ direction. Selected (c) in-plane and (d) out-of-plane spin-resolved EDCs along the $\bar{\Gamma}-\bar{M}$ direction, measured by rotating the sample azimuthal angle by $30^{\circ}$. All measurements were obtained by unpolarized He radiation at temperature of $15 \mathrm{~K}$. Spin-polarization BE- $k$ map of (e) $P_{\text {in-plane }}$ and (f) $P_{\text {out-of-plane }}$ spin components along the $\bar{\Gamma}-\bar{K}$ direction. Color strength denotes the photoemission intensity, with red and blue colors for positive and negative sign of the spin polarization, respectively, as indicated in the color bar.

\section{CONCLUSIONS}

In summary, on the basis of the angle- and spin-resolved photoelectron spectroscopy as well as DFT and $G W$ calculations, we have demonstrated that $\mathrm{Bi}_{2} \mathrm{Te}_{2} \mathrm{~S}$ in contrast to the earlier theoretical prediction $[14,15]$ is an excellent $3 \mathrm{D}$ topological insulator characterized by $\approx 400 \mathrm{meV}$ bulk band gap and topological Dirac state with Dirac point located above the valence band and below the Fermi level lying in the band gap. The gap in the nearly stoichiometric $\mathrm{Bi}_{2} \mathrm{Te}_{2} \mathrm{~S}$ compound is twice as large as in earlier studied S-rich phase. The helical spin texture and the spin polarizations of the TSS as well as deeper valence-band surface states have been revealed. The isolated Dirac cone on the surface of $\mathrm{Bi}_{2} \mathrm{Te}_{2} \mathrm{~S}$ will be particularly important for the study of topological transport properties. This phase-homogeneous ternary compound can be regarded as the model topological insulator that is promising for a variety of spintronic applications.

\section{ACKNOWLEDGMENTS}

We acknowledge partial support from the Saint Petersburg State University (Grant No. 15.61.202.2015), Tomsk State University competitiveness improvement program (Project No. 8.1.01.2017), and the Spanish Ministry of Science and Innovation (Grant No. FIS2016-75862-P). This study was partially supported by the Russian Science Foundation (Projects No. 17-12-01047, for the electrophysical properties, and No. 18-12-00169, for the theoretical calculations) and by the Russian Foundation for Basic Research (Project No. 1708-00955, for the crystal growth and structural characterization). S.V.E. acknowledges support by the Fundamental Research Program of the State Academies of Sciences for 2013-2020. I.P.R. acknowledges support by the Ministry of Education and Science of the Russian Federation within the framework of the governmental program Megagrants (state task No. 3.8895.2017/P220). A.K. was financially supported by KAKENHI Grants No. 26247064 and No. 17H06138. Calculations were performed using computational resources provided by Resource Center "Computer Center of SPbU" (http://cc.spbu.ru), and the SKIF-Cyberia supercomputer at the National Research Tomsk State University. Some of the measurements were performed at the ESPRESSO beam line under Project No. 15-A-54. E.A. acknowledges all HiSOR staff for help during the experiments.
[1] H. Zhang, C.-X. Liu, X.-L. Qi, X. Dai, Z. Fang, and S.-C. Zhang, Nat. Phys. 5, 438 (2009).

[2] M. Z. Hasan and C. L. Kane, Rev. Mod. Phys. 82, 3045 (2010).

[3] Y. Xia, D. Qian, D. Hsieh, L. Wray, A. Pal, H. Lin, A. Bansil, D. Grauer, Y. S. Hor, R. J. Cava, and M. Z. Hasan, Nat. Phys. 5, 398 (2009).

[4] Y. L. Chen, J. G. Analytis, J.-H. Chu, Z. K. Liu, S.-K. Mo, X. L. Qi, H. J. Zhang, D. H. Lu, X. Dai, Z. Fang, S. C. Zhang, I. R. Fisher, Z. Hussain, and Z.-X. Shen, Science 325, 178 (2009).

[5] D. Hsieh, Y. Xia, D. Qian, L. Wray, J. H. Dil, F. Meier, J. Osterwalder, L. Patthey, J. G. Checkelsky, N. P. Ong, A. V. Fedorov, H. Lin, A. Bansil, D. Grauer, Y. S. Hor, R. J. Cava, and M. Z. Hasan, Nat. Phys. 460, 1101 (2009).
[6] S. V. Eremeev, Y. M. Koroteev, and E. V. Chulkov, JETP Lett. 91, 387 (2010).

[7] K. Kuroda, M. Arita, K. Miyamoto, M. Ye, J. Jiang, A. Kimura, E. E. Krasovskii, E. V. Chulkov, H. Iwasawa, T. Okuda, K. Shimada, Y. Ueda, H. Namatame, and M. Taniguchi, Phys. Rev. Lett. 105, 076802 (2010).

[8] D. O. Scanlon, P. D. C. King, R. P. Singh, A. de la Torre, S. M. Walker, G. Balakrishnan, F. Baumberger, and C. R. A. Catlow, Adv. Mater. 24, 2154 (2012).

[9] N. H. Jo, K. J. Lee, C. M. Kim, K. Okamoto, A. Kimura, K. Miyamoto, T. Okuda, Y. K. Kim, Z. Lee, T. Onimaru, T. Takabatake, and M. H. Jung, Phys. Rev. B 87, 201105 (2013). 
[10] M. Neupane, S.-Y. Xu, L. A. Wray, A. Petersen, R. Shankar, N. Alidoust, C. Liu, A. Fedorov, H. Ji, J. M. Allred, Y. S. Hor, T.-R. Chang, H.-T. Jeng, H. Lin, A. Bansil, R. J. Cava, and M. Z. Hasan, Phys. Rev. B 85, 235406 (2012).

[11] Z. Ren, A. A. Taskin, S. Sasaki, K. Segawa, and Y. Ando, Phys. Rev. B 82, 241306 (2010).

[12] K. Miyamoto, A. Kimura, T. Okuda, H. Miyahara, K. Kuroda, H. Namatame, M. Taniguchi, S. V. Eremeev, T. V. Menshchikova, E. V. Chulkov, K. A. Kokh, and O. E. Tereshchenko, Phys. Rev. Lett. 109, 166802 (2012).

[13] A. M. Shikin, I. I. Klimovskikh, S. V. Eremeev, A. A. Rybkina, M. V. Rusinova, A. G. Rybkin, E. V. Zhizhin, J. Sánchez-Barriga, A. Varykhalov, I. P. Rusinov, E. V. Chulkov, K. A. Kokh, V. A. Golyashov, V. Kamyshlov, and O. E. Tereshchenko, Phys. Rev. B 89, 125416 (2014).

[14] H. Lin, T. Das, L. A. Wray, S.-Y. Xu, M. Z. Hasan, and A. Bansil, New J. Phys. 13, 095005 (2011)

[15] L.-L. Wang and D. D. Johnson, Phys. Rev. B 83, 241309 (2011).

[16] I. P. Rusinov, I. A. Nechaev, and E. V. Chulkov, JETP Lett. 98, 397 (2013).

[17] D. Harker, Z. Kristallogr. 89, 175 (1934).

[18] H. Liu, C. R. Knowles, and L. L. Y. Chang, Can. Mineral. 33, 115 (1995)

[19] N. J. Cook, C. L. Ciobanu, T. Wagner, and C. J. Stanley, Can. Mineral. 45, 665 (2007).

[20] A. C. Glatz, Am. Mineral. 52, 161 (1967).

[21] H. Ji, J. M. Allred, M. K. Fuccillo, M. E. Charles, M. Neupane, L. A. Wray, M. Z. Hasan, and R. J. Cava, Phys. Rev. B 85, 201103 (2012).

[22] K. A. Kokh, S. V. Makarenko, V. A. Golyashov, O. A. Shegai, and O. E. Tereshchenko, CrystEngComm 16, 581 (2014).

[23] T. Bathon, S. Achilli, P. Sessi, V. A. Golyashov, K. A. Kokh, O. E. Tereshchenko, and M. Bode, Adv. Mater. 28, 2183 (2016).

[24] T. Okuda, K. Miyamaoto, H. Miyahara, K. Kuroda, A. Kimura, H. Namatame, and M. Taniguchi, Rev. Sci. Instrum. 82, 103302 (2011).

[25] T. Okuda, K. Miyamoto, A. Kimura, H. Namatame, and M. Taniguchi, J. Electron Spectrosc. Relat. Phenom. 201, 23 (2015).

[26] P. E. Blöchl, Phys. Rev. B 50, 17953 (1994).

[27] G. Kresse and D. Joubert, Phys. Rev. B 59, 1758 (1999).

[28] G. Kresse and J. Hafner, Phys. Rev. B 48, 13115 (1993).

[29] G. Kresse and J. Furthmüller, Comput. Mater. Sci. 6, 15 (1996).
[30] J. P. Perdew, K. Burke, and M. Ernzerhof, Phys. Rev. Lett. 77, 3865 (1996).

[31] S. Grimme, J. Antony, S. Ehrlich, and H. Krieg, J. Chem. Phys. 132, 154104 (2010).

[32] N. Marzari and D. Vanderbilt, Phys. Rev. B 56, 12847 (1997).

[33] W. Zhang, R. Yu, H.-J. Zhang, X. Dai, and Z. Fang, New J. Phys. 12, 065013 (2010).

[34] O. V. Yazyev, E. Kioupakis, J. E. Moore, and S. G. Louie, Phys. Rev. B 85, 161101 (2012).

[35] I. A. Nechaev and E. V. Chulkov, Phys. Rev. B 88, 165135 (2013).

[36] I. A. Nechaev, I. Aguilera, V. De Renzi, A. di Bona, A. Lodi Rizzini, A. M. Mio, G. Nicotra, A. Politano, S. Scalese, Z. S. Aliev, M. B. Babanly, C. Friedrich, S. Blügel, and E. V. Chulkov, Phys. Rev. B 91, 245123 (2015).

[37] M. Michiardi, I. Aguilera, M. Bianchi, V. E. de Carvalho, L. O. Ladeira, N. G. Teixeira, E. A. Soares, C. Friedrich, S. Blügel, and P. Hofmann, Phys. Rev. B 90, 075105 (2014).

[38] I. Aguilera, C. Friedrich, G. Bihlmayer, and S. Blügel, Phys. Rev. B 88, 045206 (2013).

[39] I. A. Nechaev, R. C. Hatch, M. Bianchi, D. Guan, C. Friedrich, I. Aguilera, J. L. Mi, B. B. Iversen, S. Blügel, P. Hofmann, and E. V. Chulkov, Phys. Rev. B 87, 121111 (2013).

[40] P. D. C. King, R. C. Hatch, M. Bianchi, R. Ovsyannikov, C. Lupulescu, G. Landolt, B. Slomski, J. H. Dil, D. Guan, J. L. Mi, E. D. L. Rienks, J. Fink, A. Lindblad, S. Svensson, S. Bao, G. Balakrishnan, B. B. Iversen, J. Osterwalder, W. Eberhardt, F. Baumberger, and P. Hofmann, Phys. Rev. Lett. 107, 096802 (2011).

[41] Z.-H. Zhu, G. Levy, B. Ludbrook, C. N. Veenstra, J. A. Rosen, R. Comin, D. Wong, P. Dosanjh, A. Ubaldini, P. Syers, N. P. Butch, J. Paglione, I. S. Elfimov, and A. Damascelli, Phys. Rev. Lett. 107, 186405 (2011).

[42] M. Bahramy, P. King, A. de la Torre, J. Chang, M. Shi, L. Patthey, G. Balakrishnan, P. Hofmann, R. Arita, N. Nagaosa, and F. Baumberger, Nat. Commun. 3, 1159 (2012).

[43] L. Fu, Phys. Rev. Lett. 103, 266801 (2009).

[44] M. Nomura, S. Souma, A. Takayama, T. Sato, T. Takahashi, K. Eto, K. Segawa, and Y. Ando, Phys. Rev. B 89, 045134 (2014).

[45] C. Pauly, G. Bihlmayer, M. Liebmann, M. Grob, A. Georgi, D. Subramaniam, M. R. Scholz, J. Sánchez-Barriga, A. Varykhalov, S. Blügel, O. Rader, and M. Morgenstern, Phys. Rev. B 86, 235106 (2012). 